\title{
Overall Safety of Ospemifene in Postmenopausal Women from Placebo-Controlled Phase 2 and 3 Trials
}

\author{
James A. Simon, MD, Corrado Altomare, MD, Susannah Cort, MD, \\ Wei Jiang, $\mathrm{PhD}$, and JoAnn V. Pinkerton, $\mathrm{MD}^{3}$
}

\begin{abstract}
Objective: To evaluate the safety of daily oral ospemifene $60 \mathrm{mg}$, estrogen agonist/antagonist, used to treat moderate-tosevere dyspareunia due to postmenopausal vulvovaginal atrophy, which is part of genitourinary syndrome of menopause. Methods: Post hoc analysis of safety data (treatment-emergent adverse events [TEAEs]) pooled from six phase 2 and 3 randomized, double-blind, multicenter placebo-controlled studies, evaluating the effects of ospemifene $60 \mathrm{mg}$ on the breast, cardiovascular system, and bone in postmenopausal women.

Results: At least one TEAE was reported by $67.6 \%$ (840/1242) and $54.1 \%$ (518/958) of women taking ospemifene $60 \mathrm{mg}$ and placebo, respectively. Most TEAEs were mild or moderate and occurred within 4 to 12 weeks. The most commonly reported TEAEs with ospemifene were hot flush ( $8.5 \%$ vs. $3.3 \%$ for placebo) and urinary tract infection (6.5\% vs. $4.8 \%$ ). Discontinuation due to TEAEs was $7.6 \%$ with ospemifene and $3.8 \%$ with placebo. Most women discontinued treatment due to adverse events (AEs): hot flushes, muscle spasms, headache, and vaginal discharge. Serious AEs occurred infrequently (ospemifene, 2.6\%; placebo, 1.8\%); most were not considered related to treatment. Breast cancer and other breast-related TEAE incidences were comparable between ospemifene (2.5\%) and placebo (2.2\%), and cardiovascular TEAE incidence, including deep vein thrombosis, was low with ospemifene $(0.3 \%)$ and placebo $(0.1 \%)$.

Conclusion: No unexpected safety signals were reported, and discontinuation due to TEAEs was low, with use of ospemifene $60 \mathrm{mg}$ versus placebo in six phase 2 and 3 trials, suggesting a lack of detrimental effects on the breast, bone, and cardiovascular health of postmenopausal women when ospemifene is used to effectively treat moderate-to-severe postmenopausal dyspareunia.
\end{abstract}

Keywords: dyspareunia, ospemifene, estrogen receptor agonist and antagonist, selective estrogen receptor modulator, vulvar and vaginal atrophy

\section{Introduction}

$\mathbf{V}$ UlVAR AND VAGINAL atrophy (VVA) resulting from estrogen loss at the onset of menopause is a chronic and progressive condition affecting $\sim 45 \%$ to $57 \%$ of postmenopausal women. ${ }^{1-3}$ Considered to be a component of genitourinary symptom of menopause (GSM), ${ }^{4}$ VVA is commonly accompanied by physiological changes to the vulva and vagina (i.e., dryness or pallor of vaginal mucosa, thinning of vaginal rugae, or petechiae) and is associated with symptoms of vaginal dryness and dyspareunia. ${ }^{3}$ Vaginal dryness was recently found to be the most common menopausal symptom in North American and European women with vaginal symptoms. ${ }^{5}$ VVA symptoms are persistent, progressive, and may have an adverse effect on quality of life. ${ }^{3,6}$ Surveys of women with VVA and their partners also show that vaginal discomfort negatively impacts intimate relationships. ${ }^{7,8}$

Even though VVA is persistent and worsens in severity if left untreated, less than one-third of postmenopausal women seek treatment for their symptoms. ${ }^{9}$ Over-the-counter vaginal lubricants and moisturizers are often the first-line treatments

\footnotetext{
${ }^{1}$ Women's Health and Research Consultants, Washington, District of Columbia.

${ }^{2}$ Shionogi, Inc., Florham Park, New Jersey.

${ }^{3}$ Midlife Health Center, Department of Obstetrics and Gynecology, University of Virginia, Charlottesville, Virginia.
}

(C) James A. Simon et al. 2017; Published by Mary Ann Liebert, Inc. This is an Open Access article distributed under the terms of the Creative Commons Attribution License, which permits unrestricted use, distribution, and reproduction in any medium, provided the original work is properly cited. Mary Ann Liebert, Inc. offers reprint services for those who want to order professionally produced copies of articles published under the Creative Commons Attribution (CC BY) license. To obtain a price quote, email Reprints@liebertpub.com. Please include the article's title or DOI, quantity, and delivery destination in your email. 
for postmenopausal VVA, but they do not address the underlying atrophy due to changes in proportions of vaginal cells (decrease in superficial cells and increase in parabasal cells), and have been perceived as messy and inconvenient by many women. ${ }^{6,10}$ Hormone therapy $(\mathrm{HT})$, either vaginal or systemic, is available to women suffering from moderate-tosevere VVA or GSM. ${ }^{6}$ However, HT use has been limited by women's safety concerns associated with systemic exposure to estrogens and concerns raised by the black boxed warning required by the United States Food and Drug Administration (FDA) for systemic and vaginal estrogen therapies. ${ }^{9,11,12}$

Ospemifene is an orally administered, estrogen receptor agonist/antagonist (also known as a selective estrogen receptor modulator [SERM]) approved in the United States for the treatment of moderate-to-severe dyspareunia due to postmenopausal $\mathrm{VVA}^{13}$ and in Europe for the treatment of moderate-to-severe symptomatic VVA in postmenopausal women who are not candidates for local vaginal estrogen therapy. ${ }^{14}$ Clinical studies have shown that ospemifene, at the approved dose of $60 \mathrm{mg}$, significantly reduces the severity of dyspareunia and has a beneficial effect on vaginal dryness. ${ }^{15-21}$ Hot flushes are a commonly reported adverse event (AE) with ospemifene $60 \mathrm{mg}^{15,18,19}$; however, a recently published pooled analysis of hot flush treatment-emergent adverse events (TEAEs) found that the frequency of hot flushes declined after 4 weeks of ospemifene treatment. ${ }^{22}$ In addition, while ospemifene has been shown to have estrogenlike effects on the vaginal epithelium, it has a neutral effect on the endometrium, with no reported cases of endometrial cancer or atypical endometrial hyperplasia over a treatment period of 52 weeks. ${ }^{16}$ However, the label for ospemifene has a black box warning about estrogen agonism in the endometrium. ${ }^{13}$ A previously reported post hoc analysis of safety data also revealed that ospemifene does not have detrimental effects on the lipid and coagulation parameters in postmenopausal women. $^{23}$ Finally, preclinical and clinical data demonstrated that ospemifene $60 \mathrm{mg}$ has a beneficial estrogenic effect on bone $^{24-28}$ and antiestrogenic effect on breast tissue. ${ }^{21,25,26,28-32}$

In this study, we report results from an integrated safety analysis of pooled data from six phase 2 and 3 clinical trials evaluating the effect of ospemifene $60 \mathrm{mg}$ on the breast, cardiovascular system, and bone in postmenopausal women.

\section{Materials and Methods}

\section{Study design}

Clinical safety data were pooled from six phase 2 and 3 , double-blind placebo-controlled studies (studies 15-50717, $15-50615,{ }^{22} 1506002,{ }^{30} 15-50821,{ }^{17,18} 15-50310^{15} / 15-50310 x,{ }^{20}$ $15-50718^{21}$; Table 1$)$. The pooled population for this safety analysis had been randomized to either placebo or ospemifene $5-90 \mathrm{mg} /$ day, with the majority of ospemifene-treated women $(73.2 \%$; 1242/1696) receiving the FDA-approved dose of $60 \mathrm{mg}$. This report presents the safety data for ospemifene $60 \mathrm{mg} /$ day. The duration of treatment ranged from 6 to 52 weeks in length.

Briefly, all studies randomized participants 1:1 except for the 52-week, long-term safety study $\left(15-50718^{21}\right)$, which randomized women $6: 1$ to receive ospemifene $60 \mathrm{mg}$ or placebo. The 40-week extension study $\left(15-50310 \mathrm{x}^{20}\right)$ required participants to continue the randomized therapy they received during the previous 12 -week study $\left(15-50310^{15}\right)$.
Another 12-week study 15-50717 was an unpublished phase 2 dose-ranging study that compared the efficacy of once daily ospemifene at doses of 5,15 , and $30 \mathrm{mg}$ with placebo in postmenopausal women with VVA (since ospemifene $60 \mathrm{mg}$ is not included in study 15-50717, only placebo subjects from this study were included in the analysis reported here).

All studies were carried out in accordance with the recommendations from the Declaration of Helsinki. Study protocols, amendments, and informed consents were reviewed and approved by appropriate Institutional Review Boards or Independent Ethics Committees. All subjects from all studies provided written informed consent before study initiation.

\section{Study population}

Postmenopausal women aged 40 to 80 years with $\leq 5 \%$ vaginal superficial cells on a vaginal smear and a vaginal $\mathrm{pH}$ $>5.0$ were eligible to participate in studies $15-50717$ and $15-$ $50718 .^{21}$ Studies $15-50310^{15} / 15-50310 \mathrm{x}^{20}$ and $15-50821^{17,18}$ required women to have at least 1 self-reported, most bothersome (moderate-to-severe) symptom of VVA (i.e., dyspareunia or vaginal dryness; study $15-50310^{15}$ could include other related symptoms). Study $1506002^{30}$ was limited to postmenopausal women (45-65 years in age) with an intact uterus, and study $15-50615^{22}$ included postmenopausal women between the ages of 40 and 70 years with $\geq 7$ moderate, severe, or very severe hot flushes per day or 50 per week.

Studies $15-50821,{ }^{21} 15-50615,{ }^{22} 15-50310,{ }^{15}$ and $15-50717$ included women with and without an intact uterus, whereas studies $15-50718,{ }^{17,18} 1506002,{ }^{30}$ and $15-50310 x^{20}$ (the 40week extension study) were limited to postmenopausal women with an intact uterus.

Women with an intact uterus were required to have a double-layer endometrial thickness $<4 \mathrm{~mm}$; no evidence of hyperplasia, cancer, or other pathology based on endometrial biopsy; and a negative Papanicolaou (PAP) test at screening. All women were required to have negative mammogram and normal clinical breast examination findings at screening.

\section{Safety assessments and analysis}

All analyses comparing ospemifene $60 \mathrm{mg}$ and placebo were performed using descriptive statistics on the safety populations from placebo-controlled phase 2 and 3 studies. The safety populations were all enrolled women who took at least one dose of study drug. Numbers and percentages of women who experienced AEs were summarized. AEs were coded according to the Medical Dictionary for Regulatory Activities (MedDRA), and only TEAEs were summarized in the integrated summary. No missing data were imputed.

Adverse events. Safety assessments were descriptive in nature and included length of exposure to study medication; TEAEs and serious AEs (SAEs), deaths, and discontinuations due to AEs; and specific breast and cardiovascular safety assessments. A TEAE was defined as an AE with an onset date on or after the first dose of study medication and up to 30 days following the last dose date and was summarized by system organ class and preferred term. All TEAEs were rated by the investigator as mild, moderate, or severe and categorized by relatedness to treatment and duration of exposure. Events were considered treatment related if they were rated as possibly, probably, or definitely related, or if relatedness was missing or unknown. SAEs considered related to 


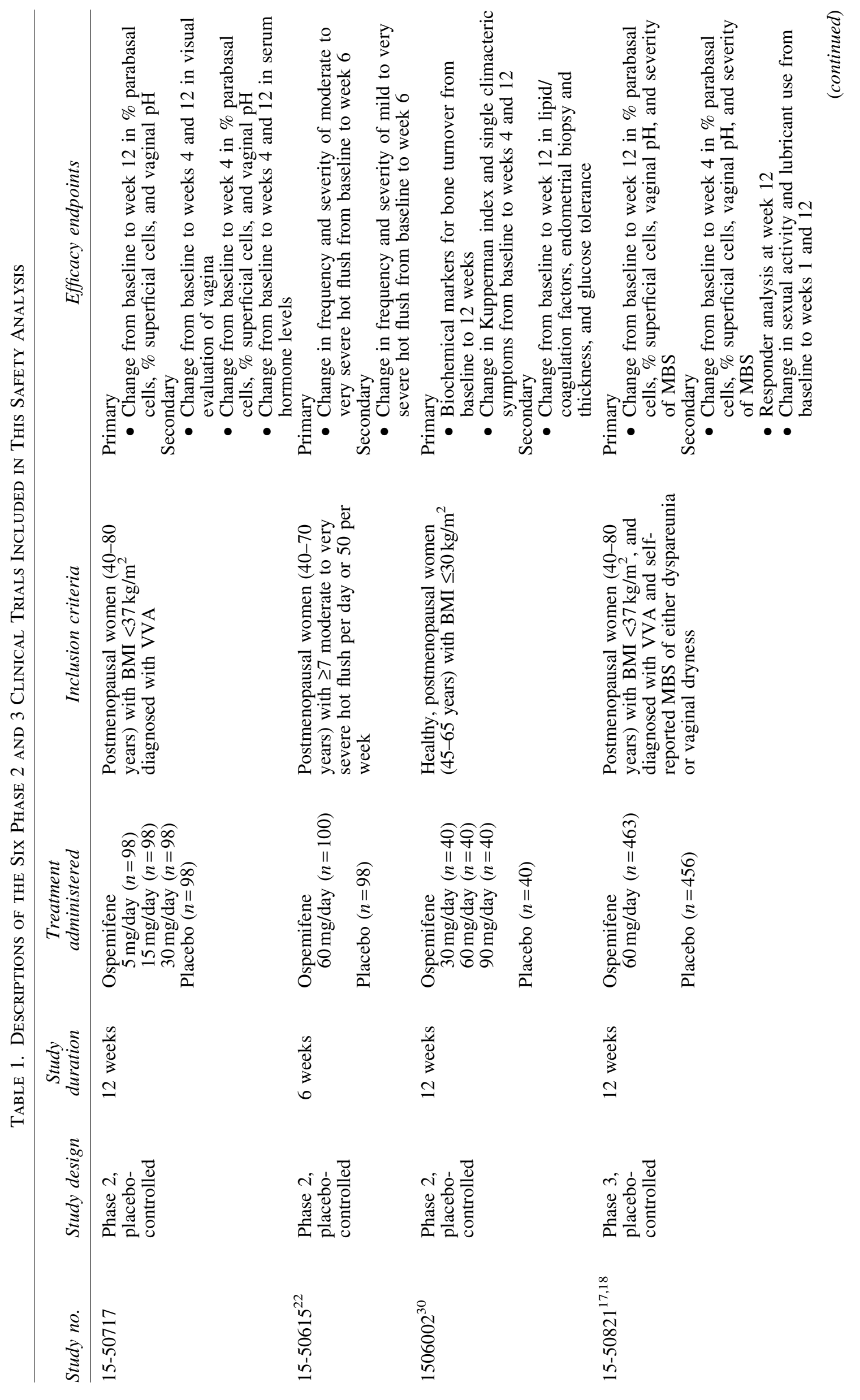




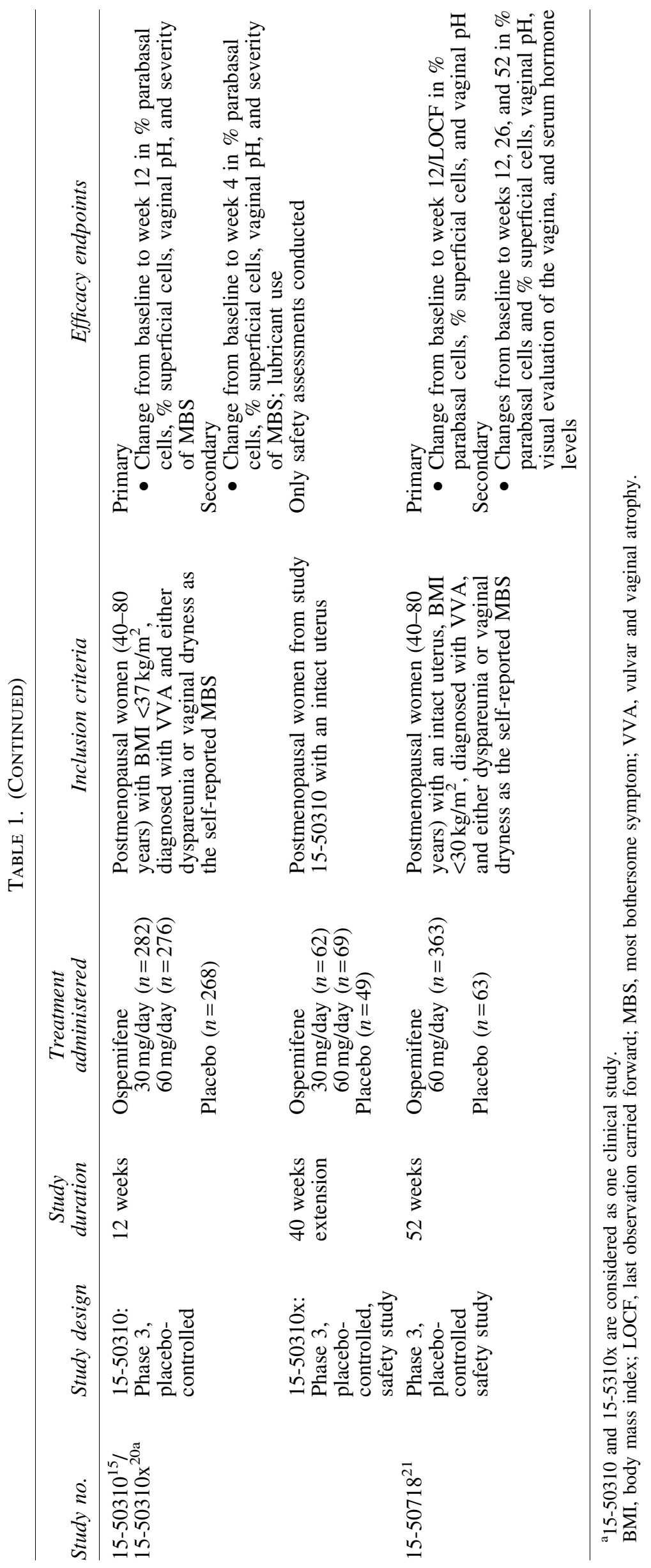


treatment were reported any time after the first dose and SAEs considered not related to treatment up to 30 days of the last dose.

Breast safety. Breast-related TEAEs were summarized by numbers and percentages of women based on a search of TEAEs in the pooled safety database by system organ class and preferred terms. Mammograms were performed at baseline and 12 months, and breast palpations were performed at baseline, 12 weeks, 6 months, and 12 months to assess breast safety. Findings on mammograms and palpations were classified by the investigator as normal, abnormal-not clinically significant, or abnormal-clinically significant. Breast density was not assessed.

Cardiovascular safety. Cardiovascular-related safety assessments included the occurrence of TEAEs related to myocardial infarction, sudden death, stroke cases, and venous thromboembolism identified based on a search of TEAEs in the pooled safety database by system organ class and preferred terms.

Vertebral and other fracture safety. Incidence of vertebral and other fractures was identified by a search of the pooled safety database for any TEAEs having a higher level group term or preferred term of fractures.

\section{Results}

\section{Patient disposition and demographics}

A total of 2200 postmenopausal women were randomized to receive ospemifene $60 \mathrm{mg}(n=1242)$ or placebo $(n=958$; Fig. 1). The median duration of exposure was similar in both groups (ospemifene, 86 days [range 1-395]; placebo, 84 days [range 1-378]). Most women randomized to ospemifene $60 \mathrm{mg}(85.4 \% ; 1061 / 1242)$ and placebo $(87.2 \% ; 835 /$ 958) completed treatment (Fig. 1). A larger percentage of ospemifene- $(7.6 \%)$ versus placebo-treated women $(3.7 \%)$ discontinued treatment due to an AE (Fig. 1).

Baseline demographics were comparable between the two treatment groups. Over $90 \%$ of the generally healthy women included in this analysis were white, with an average age of 59 years and mean body mass index (BMI) of $26 \mathrm{~kg} / \mathrm{m}^{2}$ (Table 2). Both ospemifene- $(87.2 \% ; 1083 / 1242)$ and placebo-treated $(86.1 \% ; 825 / 958)$ women took concomitant medications; most frequently, ibuprofen, acetylsalicylic acid, levothyroxine, calcium, and multivitamins (13\%-16\% each medication).

\section{Adverse events}

TEAEs and treatment-related TEAEs were reported by $67.6 \%$ and $30.4 \%$ of ospemifene-treated women and $54.1 \%$ and $16.4 \%$ of placebo-treated women, respectively. Most

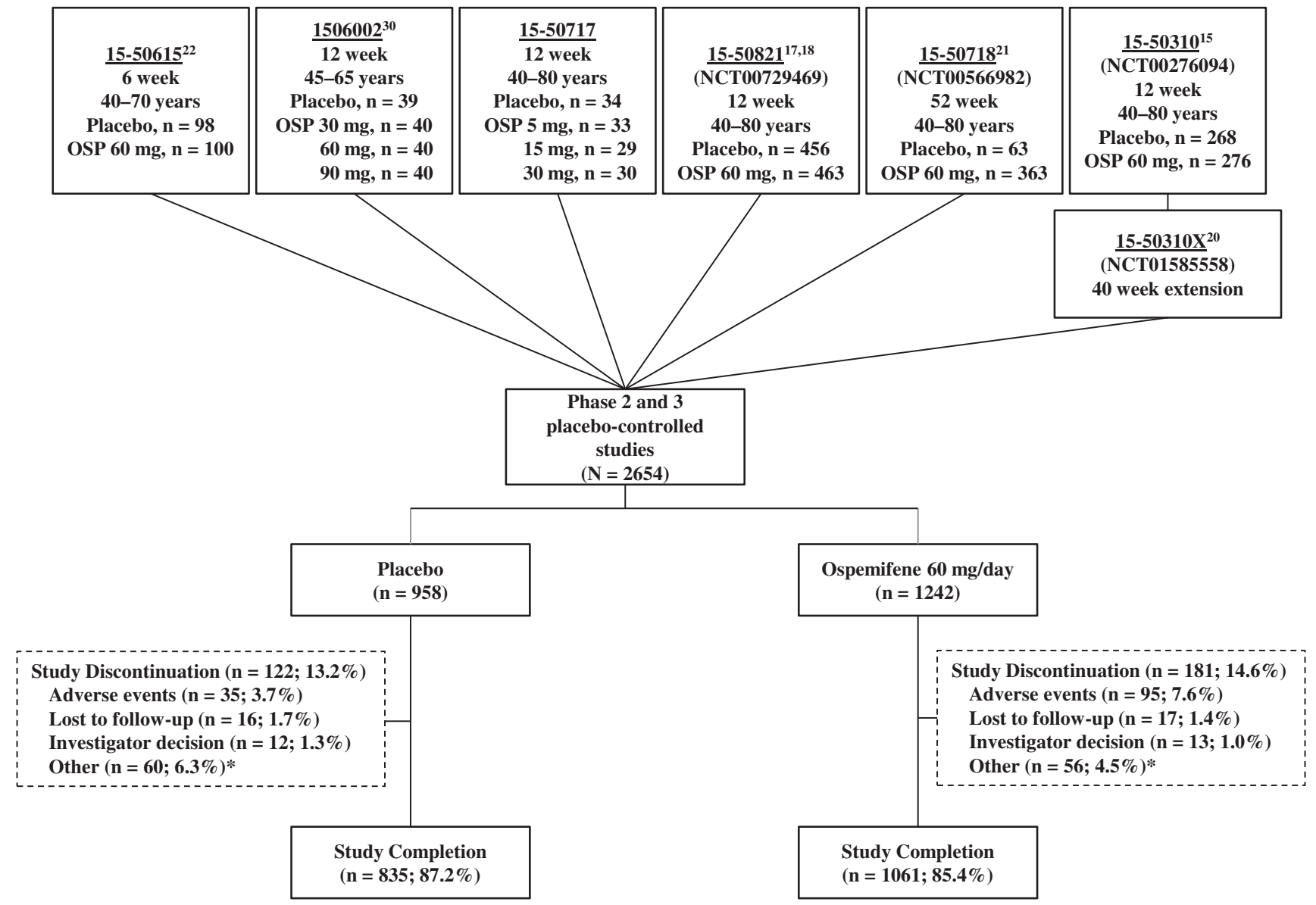

FIG. 1. Disposition of the phase 2 and 3, double-blind placebo-controlled studies included in this integrative safety analysis. 
TABle 2. Baseline Demographics AND GyneCOlOGiC Characteristics

\begin{tabular}{lcc}
\hline & & Ospemifene \\
Characteristics & Placebo & $60 m g$ \\
\hline Age, years & $n=958$ & $n=1242$ \\
Mean \pm SD & $59.1 \pm 6.27$ & $59.4 \pm 6.49$ \\
Range & $41-79$ & $40-80$ \\
$<45, n(\%)$ & $8(0.8)$ & $11(0.9)$ \\
$45-54, n(\%)$ & $212(22.1)$ & $268(21.6)$ \\
$55-64, n(\%)$ & $563(58.8)$ & $696(56.0)$ \\
$\geq 65, n(\%)$ & $175(18.3)$ & $267(21.5)$ \\
Race, $n(\%)$ & $n=956$ & $n=1242$ \\
White & $871(91.1)$ & $1159(93.3)$ \\
Black or African American & $49(5.1)$ & $47(3.8)$ \\
Asian & $9(0.9)$ & $12(1.0)$ \\
Pacific Islander & 0 & $4(0.3)$ \\
Other & $27(2.8)$ & $20(1.6)$ \\
BMI, kg/m ${ }^{2}$ & $n=958$ & $n=1242$ \\
Mean \pm SD & $26.03 \pm 4.19$ & $25.69 \pm 4.03$ \\
Range & $16.5-40.8$ & $15.7-48.6$ \\
Intact uterus & $n=957$ & $n=1242$ \\
$n(\%)$ & $570(59.6)$ & $851(68.5)$ \\
Number of pregnancies & $n=724$ & $n=739$ \\
Mean \pm SD & $2.40 \pm 1.63$ & $2.40 \pm 1.66$ \\
Vaginal birth & $n=919$ & $n=1202$ \\
$n(\%)$, Yes & $678(73.8)$ & $921(76.6)$ \\
Mean \pm SD & $1.70 \pm 1.37$ & $1.70 \pm 1.30$ \\
Previous hormone treatment, ${ }^{\text {a }}$ & $n=958$ & $n=1242$ \\
$n(\%)$, Yes & $183(19.1)$ & $262(21.1)$ \\
\hline
\end{tabular}

${ }^{\mathrm{a}}$ Including estrogen and/or progestin therapies, vaginal hormone products, hormonally active herbal therapies, and/or soy supplements within 6 months of the first dose of study drug.

$\mathrm{SD}$, standard deviation.

TEAEs and treatment-related TEAEs were mild or moderate in severity. Most TEAEs occurred within 4 to 12 weeks of study start, and the median time to onset of any TEAE was 23 days with ospemifene and 22 days with placebo.

The most commonly reported TEAEs with ospemifene $60 \mathrm{mg}$ were hot flush $(8.5 \%$ vs. $3.3 \%$ for placebo), urinary tract infection $(6.5 \%$ vs. $4.8 \%)$, headache (5.4\% vs. $5.9 \%)$, nasopharyngitis (5.4\% vs. $3.1 \%)$, muscle spasms (4.4\% vs. $1.4 \%)$, vaginal discharge ( $4.4 \%$ vs. $0.4 \%$ ), and vulvovaginal candidiasis $(4.3 \%$ vs. $0.5 \%$; Table 3 ). The most common treatment-related TEAEs with ospemifene $60 \mathrm{mg}$ were hot flush ( $7.5 \%$ vs. $2.6 \%$ for placebo), vaginal discharge (3.8\% vs. $0.3 \%)$, muscle spasms (3.2\% vs. $0.9 \%)$, and headache ( $2.4 \%$ vs. $2.4 \%$; Supplementary Table S1).

TEAEs leading to discontinuation were experienced by $7.6 \%(95 / 1242)$ of women treated with ospemifene versus $3.8 \%$ (36/958) with placebo (Supplementary Table S2). The most common TEAEs leading to discontinuation of ospemifene were hot flush $(1.0 \%, 0.3 \%$ for placebo), muscle spasms $(0.6 \%, 0.1 \%$ for placebo), headache $(0.5 \%, 0.2 \%$ for placebo), and vaginal discharge $(0.5 \%, 0 \%$ for placebo; Supplementary Table S2).

SAEs occurred with low frequency (2.6\% for ospemifene $60 \mathrm{mg}, 1.8 \%$ for placebo; Supplementary Table S3) and most were considered unrelated to treatment. Treatment-emergent SAEs reported by more than one patient treated with ospemifene $60 \mathrm{mg}$ were appendicitis $(0.2 \%)$, diverticulitis $(0.2 \%)$,
Table 3. Number and Percentage of Women RePorting THE Most Frequent ( $>2 \%$ OF WoMEN) Treatment-Emergent Adverse Events

\begin{tabular}{lcc}
\hline Preferred term, ${ }^{\mathrm{a}} \mathrm{n}(\%)$ & $\begin{array}{c}\text { Placebo } \\
(\mathrm{n}=958)\end{array}$ & $\begin{array}{c}\text { Ospemifene } \\
60 \mathrm{mg} \\
(\mathrm{n}=1242)\end{array}$ \\
\hline Any TEAE & $518(54.1)$ & $840(67.6)$ \\
Hot flush & $32(3.3)$ & $106(8.5)$ \\
Urinary tract infection & $46(4.8)$ & $81(6.5)$ \\
Headache & $57(5.9)$ & $67(5.4)$ \\
Nasopharyngitis & $30(3.1)$ & $67(5.4)$ \\
Muscle spasms & $13(1.4)$ & $55(4.4)$ \\
Vaginal discharge & $4(0.4)$ & $55(4.4)$ \\
Vulvovaginal candidiasis & $5(0.5)$ & $53(4.3)$ \\
Back pain & $23(2.4)$ & $37(3.0)$ \\
Sinusitis & $36(3.8)$ & $37(3.0)$ \\
Vulvovaginal & $5(0.5)$ & $38(3.1)$ \\
$\quad$ mycotic infection & $16(1.7)$ & $30(2.4)$ \\
Diarrhea & $24(2.5)$ & $27(2.2)$ \\
Arthralgia & $11(1.1)$ & $27(2.2)$ \\
Insomnia & $34(3.5)$ & $26(2.1)$ \\
Upper respiratory & & \\
$\quad$ tract infection & $13(1.4)$ & $25(2.0)$ \\
Bronchitis &
\end{tabular}

${ }^{\text {a }}$ Subjects with $>1$ TEAE that coded for the same preferred term were counted once for that preferred term.

TEAE, treatment-emergent adverse event.

and deep vein thrombosis (DVT) (0.2\%). No SAE was reported by more than one subject taking placebo. Treatment-related SAEs included endometrial hyperplasia (simple hyperplasia without atypia, $n=1$, probably related), cerebrovascular accident ( $n=1$, possibly related), ovarian cyst $(n=1$, possibly related), DVT ( $n=1$, probably related and $n=1$, possibly related; reported below), global amnesia ( $n=1$, possibly related), and nausea ( $n=1$, possibly related) in the ospemifene $60 \mathrm{mg}$ group, and one case of breast cancer in situ possibly related in the placebo group (reported below). No deaths were reported in any study.

\section{Breast safety}

The incidence of breast-related TEAEs was low with ospemifene $60 \mathrm{mg}(2.5 \%)$ and placebo (2.2\%; Table 4). The most commonly reported breast-related TEAEs with ospemifene were comparable to placebo; breast tenderness $(0.9 \%$ vs. $0.6 \%$ for placebo), breast mass $(0.6 \%$ vs. $0.4 \%)$, and breast pain $(0.6 \%$ vs. $0.3 \%)$. Breast enlargement was recorded as a corresponding diagnosis for one ospemifenetreated patient who underwent a preplanned elective breast reduction surgery 49 days after initial treatment. None of the breast-related TEAEs led to discontinuation with ospemifene $60 \mathrm{mg}$.

No cases of breast cancer were reported with ospemifene $60 \mathrm{mg}$. One woman from the placebo group discontinued treatment due to breast cancer diagnosis. One other woman taking placebo reported an SAE of breast cancer in situ but completed the study.

The percentages of women who had abnormal-not clinically significant-mammogram findings at baseline were $11.9 \%$ with ospemifene $60 \mathrm{mg}$ and $6.3 \%$ with placebo 
Table 4. Number and Percentage of Women with Treatment-Emergent Adverse Events Related to Breast, Cardiovascular, and Bone Health

\begin{tabular}{|c|c|c|}
\hline Preferred term, ${ }^{\mathrm{a}} \mathrm{n}(\%)$ & $\begin{array}{l}\text { Placebo } \\
(\mathrm{n}=958)\end{array}$ & $\begin{array}{c}\text { Ospemifene } \\
60 \mathrm{mg} \\
(\mathrm{n}=1242)\end{array}$ \\
\hline Any breast-related TEAE & $21(2.2)$ & $31(2.5)$ \\
\hline Breast tenderness & $6(0.6)$ & $11(0.9)$ \\
\hline Breast mass & $4(0.4)$ & $7(0.6)$ \\
\hline Breast pain & $3(0.3)$ & $7(0.6)$ \\
\hline Breast enlargement ${ }^{\mathrm{b}}$ & 0 & $2(0.2)$ \\
\hline Fibrocystic breast disease & $1(0.1)$ & $1(0.1)$ \\
\hline Breast calcifications & 0 & $1(0.1)$ \\
\hline Breast cyst & $4(0.4)$ & $1(0.1)$ \\
\hline Breast discomfort & $1(0.1)$ & $1(0.1)$ \\
\hline Breast disorder & 0 & $1(0.1)$ \\
\hline Breast prosthesis implantation & 0 & $1(0.1)$ \\
\hline Mammoplasty & 0 & $1(0.1)$ \\
\hline Biopsy breast & $1(0.1)$ & 0 \\
\hline Breast cancer & $1(0.1)$ & 0 \\
\hline Breast cancer in situ & $1(0.1)$ & 0 \\
\hline Breast discharge & $1(0.1)$ & 0 \\
\hline Mammary duct ectasia & $1(0.1)$ & 0 \\
\hline Any cardiovascular-related TEAE & $1(0.1)$ & $4(0.3)$ \\
\hline Deep vein thrombosis & 0 & $2(0.2)$ \\
\hline Cerebrovascular accident & $1(0.1)$ & $1(0.1)$ \\
\hline Cerebral hemorrhage & 0 & $1(0.1)$ \\
\hline $\begin{array}{l}\text { Any vertebral and other } \\
\text { fracture-related TEAE }\end{array}$ & $14(1.5)$ & $15(1.2)$ \\
\hline Foot fracture & $3(0.3)$ & $6(0.5)$ \\
\hline Ankle fracture & $3(0.3)$ & $2(0.2)$ \\
\hline Lower limb fracture & $2(0.2)$ & $2(0.2)$ \\
\hline Spinal fracture & 0 & $2(0.2)$ \\
\hline Upper limb fracture & 0 & $1(0.1)$ \\
\hline Wrist fracture & $1(0.1)$ & $2(0.2)$ \\
\hline Humerus fracture & 0 & $1(0.1)$ \\
\hline Hand fracture & $1(0.1)$ & 0 \\
\hline Patella fracture & $2(0.2)$ & 0 \\
\hline Rib fracture & $1(0.1)$ & 0 \\
\hline Tibia fracture & $1(0.1)$ & 0 \\
\hline
\end{tabular}

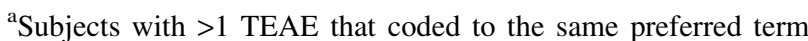
were counted once for that preferred term.

${ }^{\mathrm{b}}$ Breast enlargement reported to provide corresponding diagnosis for breast reduction; breasts had not actually enlarged during the study.

(Table 5). After 12 months, the percentage of women with abnormal—not clinically significant-findings was comparable to placebo at $7.8 \%$ with ospemifene $60 \mathrm{mg}$ and $8.5 \%$ with placebo (Table 5). No abnormal—clinically significantmammogram findings were reported. Breast density was not assessed.

Incidence of abnormal breast palpation findings at baseline was $2.8 \%$ with ospemifene $60 \mathrm{mg}$ and $2.3 \%$ for placebo (Table 5). The percentage of subjects with the abnormal finding remained low with ospemifene $60 \mathrm{mg}$ and placebo at 12 weeks, 6 months, and 12 months (Table 5).

\section{Cardiovascular safety}

The incidence of cardiovascular-related TEAEs, specifically DVT, cerebrovascular accident, and cerebral hemorrhage, was low with ospemifene (four subjects, $0.3 \%$ ) and
Table 5. Normal ANd Abnormal Breast Mammogram and Palpation Findings

\begin{tabular}{ccc}
\hline Characteristic, ${ }^{\text {a }} \mathrm{n}(\%)$ & Placebo & $\begin{array}{c}\text { Ospemifene } \\
60 \mathrm{mg}\end{array}$ \\
\hline Mammogram findings & $n=63$ & $n=363$ \\
Baseline (randomization), $n$ & 63 & 362 \\
Normal & $59(93.7)$ & $319(88.1)$ \\
Abnormal-not clinically & $4(6.3)$ & $43(11.9)$ \\
significant & & \\
Abnormal-clinical & 0 & 0 \\
$\quad$ significant & & \\
12 months, $n$ & 47 & 269 \\
Normal & $43(91.5)$ & $248(92.2)$ \\
Abnormal-not clinically & $4(8.5)$ & $21(7.8)$ \\
significant & & \\
Abnormal-clinically & 0 & 0 \\
significant & & \\
Breast palpation findings & $n=821$ & $n=1102$ \\
Baseline (randomization), $n$ & 821 & 1100 \\
Normal & $802(97.7)$ & $1069(97.2)$ \\
Abnormal & $19(2.3)$ & $31(2.8)$ \\
12 weeks, $n$ & 710 & 946 \\
Normal & $684(96.3)$ & $925(97.8)$ \\
Abnormal & $26(3.7)$ & $21(2.2)$ \\
6 months, $n$ & 96 & 379 \\
Normal & $94(97.9)$ & $377(99.5)$ \\
Abnormal & $2(2.1)$ & $2(0.5)$ \\
12 months, $n$ & 90 & 354 \\
Normal & $90(100)$ & $351(99.2)$ \\
Abnormal & 0 & $3(0.8)$ \\
\hline
\end{tabular}

${ }^{\mathrm{a}}$ Mammography and breast palpation were not performed in all studies.

placebo (one subject, 0.1\%; Table 4). Two women (one from the ospemifene $60 \mathrm{mg}$ group and one from the placebo group) experienced a cerebrovascular accident. Cerebral hemorrhage was also reported for one ospemifene-treated woman who had significant risk factors. Two ospemifene-treated patients reported DVT as a TEAE, with one patient experiencing a DVT after long-term immobilization. The second ospemifene-treated patient had entered the trial with DVT prophylaxis, suggesting a previous history of DVT. One woman from the placebo group was diagnosed with DVT after experiencing a cerebral aneurysm and subsequent cerebrovascular accident. However, this was not recorded as a TEAE as the DVT was not discovered within the prespecified treatment period (37 days after discontinuing study drug). It should be noted that all three women (two from ospemifene $60 \mathrm{mg}$ group and one from placebo group) had risk factors for DVT.

Incidence of DVT per 1000 patient years was 2.12 with ospemifene $60 \mathrm{mg}$ (two subjects) and 3.66 with placebo (one subject; reported 7 days outside of the window defined for TEAE). The hazard ratio for DVT with ospemifene $60 \mathrm{mg}$ versus placebo was 0.6 (95\% confidence interval [CI] $0.11-$ 3.14). No incidence of pulmonary embolism (PE) or retinal vein thrombosis was reported.

\section{Vertebral and other fractures}

The incidence of vertebral and other fracture-related TEAEs was low and comparable between the ospemifene 
$(1.2 \%)$ and placebo (1.5\%; Table 4) groups. Fracture-related TEAEs frequently reported by ospemifene-treated patients included foot $(n=6,0.5 \%)$, ankle $(n=3,0.3 \%)$, lower limb $(n=2,0.2 \%)$, spine $(n=2,0.2 \%)$, and wrist $(n=2,0.2 \%)$ fractures. Foot $(n=3,0.3 \%)$, ankle $(n=2,0.2 \%)$, lower limb $(n=2,0.2 \%)$, and patella $(n=2,0.2 \%)$ fractures were the most commonly reported TEAEs by placebo-treated patients. No patients discontinued the treatment due to a vertebral or other fracture-related TEAE.

\section{Discussion}

In this integrative safety analysis of pooled data from six phase 2 and 3 trials, ospemifene $60 \mathrm{mg}$ versus placebo was generally well tolerated with no unexpected safety signals on the breast, cardiovascular system, or bone of postmenopausal women. The results from this analysis further extend data from individual clinical studies demonstrating the safety of ospemifene $60 \mathrm{mg}$ in healthy postmenopausal women (study $1506002^{30}$ ), those diagnosed with VVA (studies 15$50717,15-503109 / 15-50310 x,{ }^{20} 15-50718,{ }^{21} 15-50821^{17,18}$ ), and those experiencing moderate-to-severe hot flushes (study $15-50615^{22}$ ).

Relatively few women discontinued ospemifene $60 \mathrm{mg}$ due to AEs; however, those that did reported hot flushes as the most common reason for discontinuation. A separate analysis of the pooled hot flush TEAE data found that the incidence of hot flush TEAEs was significantly greater with ospemifene $60 \mathrm{mg}$ $(8.5 \%)$ versus placebo $(3.2 \% ; p<0.0001) .{ }^{22}$ However, hot flush TEAEs were most frequently experienced within the first 4 weeks of initiating ospemifene and declined with continuous use. $^{22}$ HT use within 6 months of study start $(p=0.0294)$, longer study treatment duration $(p=0.0258)$, and higher frequency of hot flush days per month at baseline $(p=0.0326)$ were also identified as significant predictors of hot flush events with ospemifene $60 \mathrm{mg} .{ }^{22}$ Finally, ospemifene $60 \mathrm{mg}$ was not found to worsen the frequency or severity of hot flushes in a 6week, placebo-controlled study (15-50615) of 198 postmenopausal women experiencing at least 7 moderate-to-very severe hot flushes per day (or 50 per week). ${ }^{22}$

While the clinical data for breast safety (in particular breast cancer) is currently limited, this integrated safety analysis demonstrated that the breast tolerability profile (breast-related TEAEs, abnormal mammograms, and breast palpation) following ospemifene treatment for up to 52 weeks was similar to that of placebo. The clinical breast safety data for ospemifene were consistent with previously reported cell- and animalbased studies. ${ }^{28,29,32}$ A recent in vitro study found ospemifene inhibited proliferation and opposed estradiol stimulation in normal human breast tissue samples obtained from 26 postmenopausal women undergoing reduction mammoplasty. ${ }^{32}$ Collectively, the data suggest that ospemifene $60 \mathrm{mg}$ likely has neutral to antagonistic effect in the breast; however, these preclinical data need to be confirmed by longer term clinical breast safety data, including breast density data.

Ospemifene $60 \mathrm{mg}$, as shown in this analysis, does not have a detrimental effect on cardiovascular problems in healthy postmenopausal women. In addition, there were no reports of heart attacks, heart failure, or death from coronary heart disease. The cardiovascular safety of ospemifene is supported by findings from a separate post hoc analysis, which demonstrated that ospemifene did not have a negative effect on the lipid and coagulation parameters in postmenopausal women. ${ }^{23}$ Compared with placebo, ospemifene $60 \mathrm{mg}$ significantly increased high-density lipoprotein (HDL) and decreased low-density lipoprotein (LDL) levels in postmenopausal women at 3,6, and 12 months with no adverse effects on total cholesterol and triglycerides. ${ }^{23}$ Ospemifene $60 \mathrm{mg}$, compared with placebo, also significantly reduced fibrinogen levels, but the levels remained within the normal clinically acceptable range for majority of the patients. ${ }^{23}$

Reported incidences of venous thromboembolism (VTE) (a disease which includes both DVT and pulmonary embolism [PE]) events did not differ significantly between the ospemifene and placebo groups. In general, the venous thrombosis safety of ospemifene was consistent with reports for other SERMs. Incidence of VTE per 1000 patient-years was 2.12 for ospemifene $60 \mathrm{mg}, 1.7$ for bazedoxifene $20 \mathrm{mg},{ }^{33}$ and 3.5 raloxifene $60 \mathrm{mg} .{ }^{34}$ In this post hoc analysis, the likelihood of experiencing a DVT was not significant in healthy low-risk women treated with ospemifene $60 \mathrm{mg}$ (HR $0.6,95 \%$ CI $0.11-3.14$ ) compared with placebo, which is in contrast to what has been previously reported for raloxifene $60 \mathrm{mg}$ (RR 3.1, 95\% CI 1.4-6.9) ${ }^{34}$ and bazedoxifene $20 \mathrm{mg}$ (HR 8.0, 95\% CI 1.01-64.25) in older postmenopausal women with osteoporosis. ${ }^{33}$ Note, however, that this analysis was not powered to determine the risk of DVT with ospemifene or its use in higher risk populations.

Estrogen loss upon the onset of menopause often leads to loss of bone density and osteoporosis. Preclinical ${ }^{28,35}$ and individual clinical studies (using biochemical markers to evaluate bone health) ${ }^{26,27}$ have shown that ospemifene does not have a negative impact on the bone health of postmenopausal women. ${ }^{24}$ Our safety data are consistent with these previous reports as the incidence of vertebral and otherrelated fractures was low with ospemifene $60 \mathrm{mg}(1.2 \%)$ and comparable to placebo $(1.5 \%)$ in women in whom osteoporotic risk was not evaluated. Based on early clinical data, ospemifene may protect against bone loss due to menopause with continuous use; however, this needs to be confirmed by longer term clinical data on bone density and fractures in women at risk for osteoporosis.

Endometrial and uterine safety are of key importance when prescribing estrogen-based treatments, including ospemifene, to postmenopausal women. Results from a post hoc analysis of pooled phase 2 and 3 endometrial and uterine safety data for ospemifene $60 \mathrm{mg}$ have been previously reported. ${ }^{16}$ The analysis demonstrated that proportions of women with endometrial thickness $(\geq 5 \mathrm{~mm})$ and vaginal bleeding did not vary significantly with ospemifene $60 \mathrm{mg}$ $(n=851)$ versus placebo $(n=543)$ in women with an intact uterus. ${ }^{16}$ Incidence of suspected endometrial polyps based on histology was similar with ospemifene $60 \mathrm{mg}(1.4 \%)$ and placebo (1.6\%). ${ }^{16}$ Incidence of uterine polyps was $0.6 \%$ with ospemifene $60 \mathrm{mg}$ and $0.2 \%$ for placebo. ${ }^{16}$ One ospemifenetreated patient $(0.3 \%)$ experienced simple endometrial hyperplasia without atypia, which is well within the FDA recommended rate of $<1 \%$ with an upper bound $<4 \%$ of the one-side $95 \%$ CI. ${ }^{16}$ No cases of endometrial cancer, endometrial carcinomas, complex hyperplasia, or hyperplasia with atypia were observed with either ospemifene $60 \mathrm{mg}$ or placebo. ${ }^{16}$

Despite the safety data presented above, the prescribing information for ospemifene includes a boxed warning issued by the FDA, which states that "there is an increased risk of 
endometrial cancer in a woman with a uterus who uses unopposed estrogens." "13 The warning also recommends prescribing progestin when recommending an estrogen-based treatment to postmenopausal women with an intact uterus. However, this is not clinically recommended as there are no safety and efficacy data for ospemifene and progestogen combination therapy. The boxed warnings appear to be based on endometrial safety data for systemic HT and to raise caution. ${ }^{36,37}$ Women who develop postmenopausal vaginal bleeding while taking ospemifene should be evaluated with appropriate testing such as vaginal ultrasound and/or endometrial biopsy.

The strengths of this pooled analysis are the large number of subjects, the 40-week extension study, and the ability to detect the occurrence of infrequent AEs. The post hoc nature of this analysis, which combines multiple studies varying in length and endpoints, as well as the predominantly healthy, nonobese white patient population, limits the generalizability of the findings to the general population. Furthermore, this analysis of safety data combined from multiple studies was not designed or powered to rigorously determine the risk of breast, cardiovascular, and bone-related AEs. Longer term clinical trials and monitoring are needed to establish the risk of complications that typically evolve more slowly, such as endometrial cancer, breast cancer, and bone fractures, risks in different ethnic populations, or in women at risk for serious outcomes.

\section{Conclusion}

This post hoc analysis of pooled safety data for predominantly healthy, nonobese, white postmenopausal women suggests that ospemifene $60 \mathrm{mg}$ once daily does not detrimentally impact the breast, bone, and cardiovascular health of these women with results comparable to placebo. These data further extend the safety data reported by individual clinical studies and other post hoc analyses and provide valuable, clinically relevant safety information that enables healthcare providers to appropriately prescribe ospemifene $60 \mathrm{mg}$ to postmenopausal women with painful intercourse and VVA.

\section{Acknowledgments}

Medical writing support was provided by Disha Patel, $\mathrm{PhD}$, and Kathleen Ohleth, $\mathrm{PhD}$, at Precise Publications, LLC, which was funded by Shionogi, Inc. Funding: Hormos Medical Corp and Shionogi, Inc. sponsored the phase 2 and 3 trials.

\section{Author Disclosure Statement}

Dr. Simon has served (within the last year) or is currently serving as a consultant to or on the advisory boards of AbbVie, Allergan, AMAG, Amgen, Inc., Apotex, Inc., Ascend Therapeutics, Azure Biotech, JDS Therapeutics, Merck \& Co, Millendo Therapeutics, Noven, Novo Nordisk, Nuelle, Perrigo Company, PLC, Radius Health, Regeneron Pharmaceuticals, Roivant Sciences, Sanofi SA, Sebela Pharmaceuticals, Sermonix Pharmaceuticals, Shionogi, Inc., Sprout Pharmaceuticals, Symbiotec Pharmalab, TherapeuticsMD, and Valeant Pharmaceuticals; and has received (within the last year) or is currently receiving grant/research support from AbbVie, Actavis, PLC, Agile Therapeutics, Bayer Healthcare, GlaxoSmithKline, New England Research In- stitute, Novo Nordisk, Palatin Technologies, Symbio Research, and TherapeuticsMD; and has also served (within the last year) or is currently serving on the speaker's bureaus of Amgen, Eisai, Merck, Noven Pharmaceuticals, Novo Nordisk, Shionogi, Inc., and Valeant Pharmaceuticals; and is a stockholder (direct purchase) in Sermonix Pharmaceuticals. Drs. Altomare, Cort, and Jiang are employees of Shionogi, Inc. Dr. Pinkerton has served as a consultant (fees paid to the University of Virginia) for Pfizer, Inc., and in-kind editorial support from Pfizer, Noven Pharmaceuticals, Inc., TherapeuticsMD, and Shionogi, Inc.; has received grants/research support (fees paid to the University of Virginia) from TherapeuticsMD.

\section{References}

1. Santoro N, Komi J. Prevalence and impact of vaginal symptoms among postmenopausal women. J Sex Med 2009; 6:2133-2142.

2. Levine KB, Williams RE, Hartmann KE. Vulvovaginal atrophy is strongly associated with female sexual dysfunction among sexually active postmenopausal women. Menopause 2008;15:661-666.

3. Mac Bride MB, Rhodes DJ, Shuster LT. Vulvovaginal atrophy. Mayo Clin Proc 2010;85:87-94.

4. Portman DJ, Gass ML. Genitourinary syndrome of menopause: New terminology for vulvovaginal atrophy from the International Society for the Study of Women's Sexual Health and the North American Menopause Society. Menopause 2014;21:1063-1068.

5. Minkin MJ, Reiter S, Maamari R. Prevalence of postmenopausal symptoms in North America and Europe. Menopause 2015;22:1231-1238.

6. Management of symptomatic vulvovaginal atrophy: 2013 position statement of The North American Menopause Society. Menopause 2013;20:888-902.

7. Simon JA, Nappi RE, Kingsberg SA, Maamari R, Brown V. Clarifying Vaginal Atrophy's Impact on Sex and Relationships (CLOSER) survey: Emotional and physical impact of vaginal discomfort on North American postmenopausal women and their partners. Menopause 2014;21: 137-142.

8. Nappi RE, Mattsson LA, Lachowsky M, Maamari R, Giraldi A. The CLOSER survey: Impact of postmenopausal vaginal discomfort on relationships between women and their partners in Northern and Southern Europe. Maturitas 2013;75:373-379.

9. Nappi RE, Kokot-Kierepa M. Vaginal health: Insights, views \& attitudes (VIVA)-results from an international survey. Climacteric 2012;15:36-44.

10. Sinha A, Ewies A. Non-hormonal topical treatment of vulvovaginal atrophy: An up-to-date overview. Climacteric 2013;16:305-312.

11. Simon JA, Kokot-Kierepa M, Goldstein J, Nappi RE. Vaginal health in the United States: Results from the vaginal health: Insights, views \& attitudes survey. Menopause 2013; 20:1043-1048.

12. Manson JE, Goldstein SR, Kagan R, et al. Why the product labeling for low-dose vaginal estrogen should be changed. Menopause 2014;21:911-916.

13. Osphena ${ }^{\circledR}$ [(ospemifene) tablets, for oral use]. Prescribing Information. Florham Park, NJ: Shionogi, Inc., 2013.

14. Senshio (60 mg film-coated tablets). Ospemifene Summary of Product Characteristics. London, UK: Shionogi, Inc., 2015. 
15. Bachmann GA, Komi JO. Ospemifene effectively treats vulvovaginal atrophy in postmenopausal women: Results from a pivotal phase 3 study. Menopause 2010;17:480-486.

16. Constantine GD, Goldstein SR, Archer DF. Endometrial safety of ospemifene: Results of the phase 2/3 clinical development program. Menopause 2015;22:36-43.

17. Portman D, Palacios S, Nappi RE, Mueck AO. Ospemifene, a non-oestrogen selective oestrogen receptor modulator for the treatment of vaginal dryness associated with postmenopausal vulvar and vaginal atrophy: A randomised, placebocontrolled, phase III trial. Maturitas 2014;78:91-98.

18. Portman DJ, Bachmann GA, Simon JA. Ospemifene, a novel selective estrogen receptor modulator for treating dyspareunia associated with postmenopausal vulvar and vaginal atrophy. Menopause 2013;20:623-630.

19. Simon J, Portman D, Mabey RG, Jr. Long-term safety of ospemifene (52-week extension) in the treatment of vulvar and vaginal atrophy in hysterectomized postmenopausal women. Maturitas 2014;77:274-281.

20. Simon JA, Lin VH, Radovich C, Bachmann GA, Group tOS. One-year long-term safety extension study of ospemifene for the treatment of vulvar and vaginal atrophy in postmenopausal women with a uterus. Menopause 2013;20:418-427.

21. Goldstein SR, Bachmann GA, Koninckx PR, Lin VH, Portman DJ, Ylikorkala O. Ospemifene 12-month safety and efficacy in postmenopausal women with vulvar and vaginal atrophy. Climacteric 2014;17:173-182.

22. Constantine G, Archer DF, Pollycove R, Jiang W, Altomare C, Pinkerton J. Effects of ospemifene on vasomotor symptoms in phase 2 and 3 clinical trials. Menopause 2016; 23:957-964.

23. Archer DF, Altomare C, Jiang W, Cort S. Ospemifene's effects on lipids and coagulation factors: A post hoc analysis of phase 2 and 3 clinical trial data. Menopause 2017; 24:1167-1174.

24. Constantine GD, Kagan R, Miller PD. Effects of ospemifene on bone parameters including clinical biomarkers in postmenopausal women. Menopause 2016;23:638-644.

25. Kangas L, Harkonen P, Vaananen K, Peng Z. Effects of the selective estrogen receptor modulator ospemifene on bone in rats. Horm Metab Res 2014;46:27-35.

26. Komi J, Heikkinen J, Rutanen EM, Halonen K, Lammintausta R, Ylikorkala O. Effects of ospemifene, a novel SERM, on biochemical markers of bone turnover in healthy postmenopausal women. Gynecol Endocrinol 2004;18:152-158.

27. Komi J, Lankinen KS, DeGregorio M, et al. Effects of ospemifene and raloxifene on biochemical markers of bone turnover in postmenopausal women. $\mathrm{J}$ Bone Miner Metab 2006;24:314-318.

28. Soe LH, Wurz GT, Kao CJ, Degregorio MW. Ospemifene for the treatment of dyspareunia associated with vulvar and vaginal atrophy: Potential benefits in bone and breast. Int $\mathbf{J}$ Womens Health 2013;5:605-611.

29. Kangas L, Harkonen P, Vaananen K, Keskitalo J, Eigeliene N. Effects of ospemifene on breast tissue morphology and proliferation: A comparative study versus other selective estrogen receptor modulators in ovariectomized rats. Horm Metab Res 2014;46:328-332.

30. Rutanen EM, Heikkinen J, Halonen K, Komi J, Lammintausta R, Ylikorkala O. Effects of ospemifene, a novel SERM, on hormones, genital tract, climacteric symptoms, and quality of life in postmenopausal women: A doubleblind, randomized trial. Menopause 2003;10:433-439.

31. Ylikorkala O, Cacciatore B, Halonen K, et al. Effects of ospemifene, a novel SERM, on vascular markers and function in healthy, postmenopausal women. Menopause 2003; 10:440-447.

32. Eigeliene N, Kangas L, Hellmer C, Kauko T, Erkkola R, Härkönen $\mathrm{P}$. Effects of ospemifene, a novel selective estrogen-receptor modulator, on human breast tissue ex vivo. Menopause 2016;23:719-730.

33. Christiansen C, Chesnut Iii CH, Adachi JD, et al. Safety of bazedoxifene in a randomized, double-blind, placebo- and active-controlled phase 3 study of postmenopausal women with osteoporosis. BMC Musculoskelet Disord 2010;11:130.

34. Grady D, Ettinger B, Moscarelli E, et al. Safety and adverse effects associated with raloxifene: Multiple outcomes of raloxifene evaluation. Obstet Gynecol 2004;104:837-844.

35. Kangas L, Unkila M. Tissue selectivity of ospemifene: Pharmacologic profile and clinical implications. Steroids 2013;78:1273-1280.

36. Goldstein SR. Postmenopausal dyspareunia: Has the Food and Drug Administration really helped? Menopause 2013; 20:596-597.

37. Paton D. Ospemifene for the treatment of dyspareunia in postmenopausal women. Drugs Today 2014;50:357.

Address correspondence to: James A. Simon, MD Women's Health and Research Consultants 1850M Street, NW, Suite 450 Washington, DC 20036

E-mail: jsimon@jamesasimonmd.com 